\title{
Material prediction from confocal images of lasered samples
}

Hongbin Choi ${ }^{1}$, Adrian Phoulady ${ }^{2}$, Nicholas May ${ }^{3}$, Sina Shahbazmohamadi ${ }^{1}$ and Pouya Tavousi ${ }^{4}$

${ }^{1}$ University of Connecticut, Storrs, Connecticut, United States, ${ }^{2}$ REFINE Center, University of Connecticut, United States, ${ }^{3}$ University of Connecticut, Connecticut, United States, ${ }^{4}$ UConn Tech Park, University of Connecticut, storrs, Connecticut, United States

Introduction: Effective use of laser for fine-machining of material requires fine-tuning of laser-machining parameters. Based on the machining requirement and the composition of the material that needs to be ablated, proper lasering/scanning parameters must be practiced to achieve satisfactory results. Nevertheless, oftentimes, a priori accurate information about the material composition of sample of interest is not at hand and thus the material composition must be inferred during the laser-machining process. Non-trial-and-error existing methods that could be used for this purpose include energy dispersive spectroscopy (EDS) and laser induced breakdown spectroscopy (LIBS). The complexities associated with integrating such techniques with laser machining often acts as a prohibitive factor on the way of using them. Herein, we report on the development of a new technique that can predict material composition while laser machining is taking place using confocal images that have been obtained from the surface of lasered samples together with a knowledge of the lasering parameters. A multilayer fully connected neural network was trained, using a training data set, to predict the material composition of samples, within a set of unseen data, that have undergone laser machining, followed by confocal imaging. Note that, although lasering must start before material composition can be detected - which is also the case for LIBS - the amount of lasering that is needed for this purpose is minimal.

Data: Our dataset consisted of 1679 confocal images obtained from lasered samples of 3 different types, namely Aluminum, Silicon, and Copper. Lasering was performed using a femtosecond laser source and a galvo-scanning system. The lasering and scanning parameters that were varied included energy per pulse (EPP), repetition rate, number of lasering cycles, overlap between laser spots in $\mathrm{x}$ and $\mathrm{y}$ directions, and scanning pattern. The 1679 data points included redundant experiments that were performed to examine the level of consistency between repeats. Excluding the redundant data resulted in 700 unique experiments, with various material types as well as various lasering and scanning parameters. Several surface metrics were calculated from the confocal images using Mountains Lab software, out of which two metrics namely, the depth of cut (DOC) and roughness (Sq), were used as input parameters to the machine learning (ML) algorithm. Therefore, the input of each training data point consisted of the lasering and scanning parameters used for lasering the surface as well as the surface metrics extracted from the confocal images of the lasered surfaces. The output of each training data point reflected the material type of the lasered sample.

Training: We trained a four-layer fully connected neural network to predict the material type from the lasering/scanning parameters and surface metrics. In doing so, the scanning pattern feature was coded using integer numbers 1 through 6 , respectively representing bidirectional lines, cross, multiangle $\left(45^{\circ}\right)$, dot, hexagon, and contour patterns. The material type was coded by numbers 1, 2, and 3 respectively representing Aluminum, Silicon, and Copper. For the rest of the features, the real values of the quantities were used without normalization. ReLU activation function was used in all layers except for the last layer where Softmax was used instead. The dataset was randomly split into two subsets, where \%80 was selected for training and the rest for testing. The network was trained for 1000 epochs. The trained network was then used for making predictions on the test data set. 
Results: The results from one run are as follows. Accuracies of 94\%, 100\%, 100\% with 70, 383, 103 correct material predictions respectively out of 74, 383, 103 (respectively corresponding to Aluminum, Silicon, and Copper) were achieved for the training data. These accuracies for the test data were $75 \%$, $96 \%, 100 \%$ with 12, 92, 29 correct material predictions out of 16, 95, 29 samples respectively. Given that the material prediction was conducted based on selecting the greatest number among the probability values that the network reports for different material types, these probability values were further studied for the cases that a misprediction was made by the network. These probabilities are reported in Table 1. Given that all of the mispredictions are in the form of a mix up between Aluminum and Silicon, a potential hypothesis that must be further studied is that the reason for such mix up can be the close atomic numbers of Silicon and Aluminum which are 14 and 13 respectively, which can potentially result in their similar behavior in interaction with laser.

Future work: In the current work, along with the lasering/scanning parameters that are set by the user, we used two parameters of DOC and Sq, extracted from the confocal images of the lasered surfaces, to predict the sample's material type. However, using the entire image instead of these derived parameters can potentially reveal more information about the surface and thus more accurate predictions, which is the subject of our future work.

\begin{tabular}{lccc}
\hline Material & $\boldsymbol{p}_{\text {Al }}$ & $\boldsymbol{p}_{\text {Si }}$ & $\boldsymbol{p}_{\mathbf{C u}}$ \\
\hline Al & .48 & .52 & .00 \\
Al & .00 & 1.0 & .00 \\
Al & .00 & 1.0 & .00 \\
Al & .00 & 1.0 & .00 \\
Si & .99 & .01 & .00 \\
Si & .99 & .01 & .00 \\
Si & .90 & .10 & .00 \\
\hline
\end{tabular}

Figure 1. Mispredictions and corresponding probabilities

\section{References}

May, Nicholas, et al. "Correlative microscopy workflow for precise targeted failure analysis of multi-layer ceramic capacitors." Microelectronics Reliability 114 (2020): 113858.

Konnik, Matthew, et al. "Training AI-Based Feature Extraction Algorithms, for Micro CT Images, Using Synthesized Data." Journal of Nondestructive Evaluation 40.1 (2021): 1-13. 